\title{
Selection of theories, models and frameworks (TMFs) in the implementation of Healthy Eating Interventions in Centre-Based Childcare
}

\section{Abstract}

Childcare settings are ideal for health promotion; however, they may not provide food environments that support children's healthy eating behaviours. To address this problem, researchers and practitioners have designed interventions with the aid of Theories, Models and Frameworks (TMFs) with the aim to improve centre-based childcare food environments. Considering the many TMFs that exist, the question of which TMF to select remains a challenge. As such, one of the objectives of our scoping review was to explore the selection criteria used in studies that aimed to promote healthy eating in centre-based childcare.

We utilized the scoping review steps outlined by Arksey \& O’Malley (2005), and Levac, Colquhoun, \& O’Brien (2012). We searched Medline, Embase, CINAHL, ERIC, PsycINFO, Scopus, CAB Abstracts, Agricola, ProQuest Dissertations and Theses, Prospero, and Cochrane library of SR for studies published from January 1990 to October 2018. We also consulted subject matter experts and hand searched reference lists. First level screening was completed by two reviewers (Kappa $>0.8$ ). Second level was completed by one reviewer and questions were addressed through discussion with a second reviewer. TMF categorisation (i.e., individual, community, process and evaluation-focused) was informed by Nilsen (2015) and Rimer (2005). We used direct content analysis to organize the reported criteria used by authors to select TMFs. The rationales presented by authors were coded using Birken et al. (2017) criteria and definitions, which were based on seminal articles and an iterative consensus process. The categorization was completed by one reviewer (MV) and verified by a second reviewer (AF).

We retained 38 studies and identified 28 TMFs. TMFs were used to design implementation strategies, steps and outcomes, identify implementation factors and stakeholders, explain findings, and provide recommendations. Of the total of 14 criteria for selecting TMFs identified, the most frequently used were for describing a change process $(n=12 ; 23 \%)$, providing process guidance $(n=8$; $15 \%)$, and TMFs use in empirical studies $(n=7 ; 13 \%)$.

TMF selection was most often based on descriptive rather than predictive or explanatory characteristics. Increasing childcare researchers' and practitioners' awareness of existing TMFs and tools to guide TMF selection might reduce selection based on convenience or where limited evidence for TMF effectiveness exist.

\section{Conflict of Interest}

There is no conflict of interest 